D. C. Damin', M. Caetano', M. A. Rosito', C. Tarta ${ }^{1}$, P. Contu' ${ }^{1}$, P. Mancopes ${ }^{1}$,

\section{Meurer $^{2}$}

${ }^{1}$ Department of Colorectal Surgery, Hospital de Clinicas de Porto Alegre, Federal University of Rio Grande do Sul, Porto Alegre, Brazil

${ }^{2}$ Department of Pathology, Hospital de Clinicas de Porto Alegre, Federal University of Rio Grande do Sul, Porto Alegre, Brazil

\section{Corresponding Author}

\section{C. Damin, M.D.}

Av. Nilo Peçanha 2110/902

91.330.002 Porto Alegre - RS

Brazil

Fax: $\quad+55-51-33285168$

E-mail: damin@terra.com.br

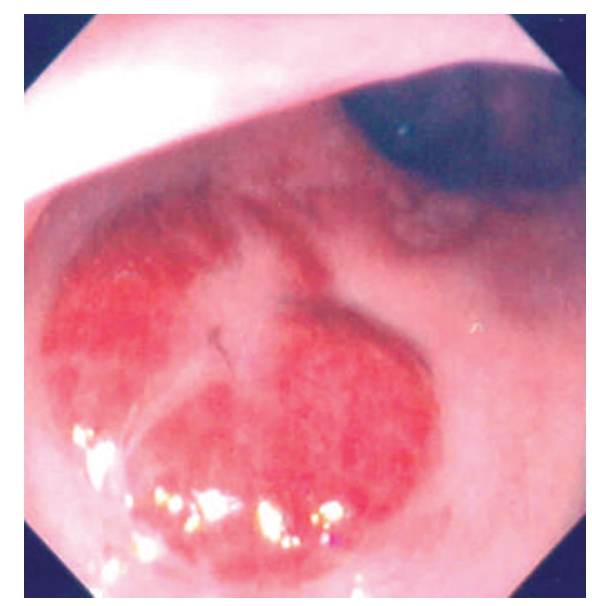

Figure 1 A 31-year-old HIV-positive man presented with diarrhea and anal bleeding. Colonoscopy revealed multiple patchy erythematous lesions located in the rectum, along with linear erosions.

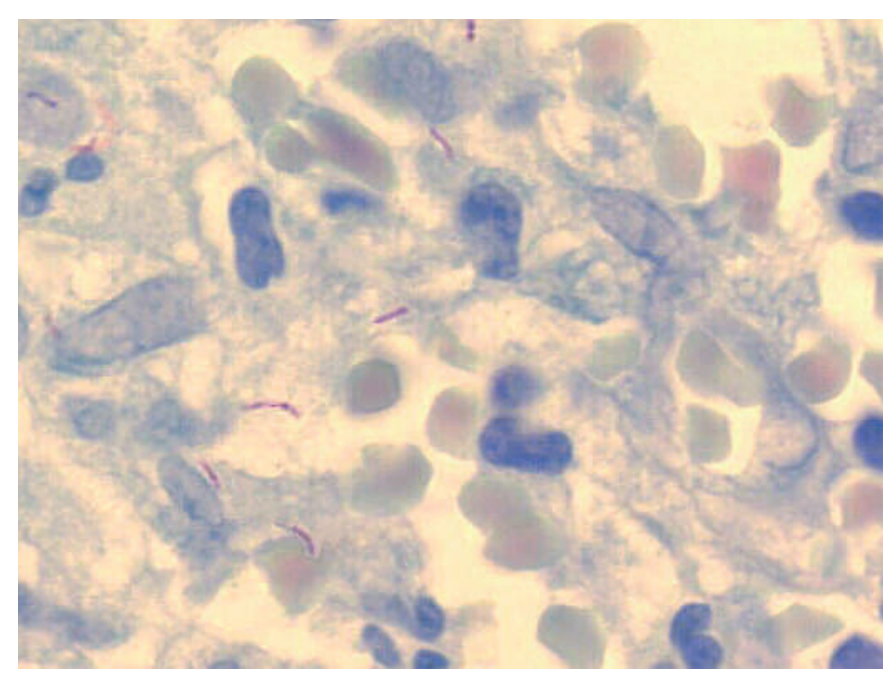

Figure 2 Histopathological analysis of rectal biopsies demonstrated the presence of innumerable free and intracellular acid-fast bacilli $(\times 32)$. Mycobacterium other than tuberculosis was cultured from the rectal specimens, confirming the diagnosis of atypical mycobacterial rectitis. The patient was treated with a combination of antimycobacterial drugs, with complete resolution of his clinical and endoscopic manifestations. 